\title{
The ophthalmic surgical backlog associated with the COVID-19 pandemic: a population-based and microsimulation modelling study
}

\author{
Tina Felfeli MD, Raphael Ximenes PhD, David M.J. Naimark MD, Philip L. Hooper MD, \\ Robert J. Campbell MD, Sherif R. El-Defrawy MD, Beate Sander PhD
}

Abstract

Background: Jurisdictions worldwide ramped down ophthalmic surgeries to mitigate the effects of COVID-19, creating a global surgical backlog. We sought to predict the long-term impact of COVID-19 on the timely delivery of non-emergent ophthalmology subspecialty surgical care in Ontario.

Methods: This is a microsimulation modelling study. We used provincial population-based administrative data from the Wait Time Information System database in Ontario for January 2019 to May 2021 and facility-level data for March 2018 to May 2021 to estimate the backlog size and wait times associated with the COVID-19 pandemic. For the postpandemic recovery phase, we estimated the resources required to clear the backlog of patients accumulated on the wait-list during the pandemic. Outcomes were accrued over a time horizon of 3 years.

Results: A total of 56923 patients were on the wait-list in the province of Ontario awaiting non-emergency ophthalmic surgery as of Mar. 15, 2020. The number of non-emergency surgeries performed in the province decreased by $97 \%$ in May 2020 and by $80 \%$ in May 2021 compared with the same months in 2019. By 2 years and 3 years since the start of the pandemic, the overall estimated number of patients awaiting surgery grew by $129 \%$ and $150 \%$, respectively. The estimated mean wait time for patients for all subspecialty surgeries increased to 282 (standard deviation [SD] 91) days in March 2023 compared with 94 (SD 97 ) days in 2019. The provincial monthly additional resources required to clear the backlog by March 2023 was estimated to be a $34 \%$ escalation from the prepandemic volumes (4626 additional surgeries).

Interpretation: The estimates from this microsimulation modelling study suggest that the magnitude of the ophthalmic surgical backlog from the COVID-19 pandemic has important implications for the recovery phase. This model can be adapted to other jurisdictions to assist with recovery planning for vision-saving surgeries.

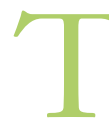
he spread of SARS-CoV-2 has led to major disruption of elective or nonurgent surgical procedures globally. ${ }^{1-4}$ Across 190 countries, it is estimated that more than 28 million surgeries were postponed in the immediate months after the COVID-19 shutdowns. ${ }^{3}$ Starting Mar. 15, 2020, in Ontario, Canada, hospitals began reducing the number of scheduled surgeries and procedures, including ophthalmic surgeries. ${ }^{5}$ Large backlogs of patients accrued as a consequence of the lockdown, which may lead to deteriorating quality of life and development of irreversible vision impairment. With the resumption of elective activities, patients are likely to be prioritized by clinical urgency, which may further lengthen delays for patients with progressive eye conditions that are not imminently vision-threatening. 6,7

Predicting the long-term backlog created for ophthalmic surgeries as a result of the COVID-19 pandemic will provide guidance for health care systems to prepare for the ongoing pandemic and postpandemic recovery phase. Microsimulation modelling offers the ability to track individual patients as they traverse the hospital system and to run scenarios to assess the impact of the reductions in ophthalmic surgeries on wait times and consequences of delayed surgeries; this can be done while taking into account the interactions between prioritization of surgeries performed based on urgency, specialty availability of resources and incidence case growth. ${ }^{8-10}$

\section{Competing interests: None declared.}

This article has been peer reviewed.

Correspondence to: Tina Felfeli, tina.felfeli@mail.utoronto.ca CMAJ Open 2021 November 23. DOI:10.9778/cmajo.20210145 
With the implementation of the provincial Wait Time Strategy database from Ontario Health, all wait times for patients are comprehensively captured for the Ontario population of 14.7 million. ${ }^{11}$ The Wait Time Information System (WTIS) database provides an opportunity to study the impact of the COVID-19 pandemic on the wait time for various subspecialty surgeries including cataract, vitreoretinal, glaucoma, cornea, oculoplastics and strabismus surgeries. ${ }^{12}$

Herein, we present a microsimulation model informed by a province-wide database, which aims to project the long-term impact of the COVID-19 pandemic on ophthalmology surgical volumes, wait times and postpandemic recovery phase in Ontario, Canada.

\section{Methods}

\section{Study design}

We developed an individual-level, discrete-time, microsimulation model. We followed Canadian Agency for Drugs and Technologies in Health (CADTH $)^{13}$ and Consolidated Health Economic Evaluation Reporting Standards (CHEERS) ${ }^{14}$ for conducting the study and reporting of its outcomes.

\section{Setting, study population and outcomes}

We set up the model in the setting of Ontario, Canada, to simulate adult patients ( $\geq 18 \mathrm{yr}$ ) waiting for ophthalmic surgery at the start of the COVID-19 pandemic. The primary outcomes of the study were the number of patients awaiting non-emergency ophthalmic surgery per month and the time to surgery (number of days on the wait-list) based on the subspecialty surgery type and level of urgency. In addition, we estimated the escalation in resources required to clear the backlog during the postpandemic recovery phase.

\section{Data sources}

We used provincial administrative data from Jan. 1, 2019, to May 31, 2021, from the Ontario WTIS database to parameterize the model. Ontario Health (formerly Cancer Care Ontario) is authorized to collect population-level data for the purpose of monitoring allocation of resources and delivery of services. The database captures data on patients who are on the wait-list queue for non-emergency surgical procedures as of the first day of each month, as well as the number of new cases added to the wait-list and number of completed surgeries performed..$^{15}$ Additionally, the database captures the wait time in days (mean and standard deviation $[\mathrm{SD}]$ ) for patients on the wait-list.

All surgical cases in Ontario are standardized into priority levels 1 to 4 (for ophthalmic surgery, 1 being the most urgent and irreversible causes of vision loss) with associated maximum surgical wait time targets. These priority-level definitions for wait times reflect the need to accelerate care that minimizes the impact of disability on patients and are accepted by the federal, provincial and territorial ministers of health (Appendix 1, Supplemental Table 1, available at www. cmajopen.ca/content/9/4/E1063/suppl/DC1). ${ }^{11}$ Given the urgency of priority 1 cases, they are not added to a wait-list and thus not captured in the Ontario WTIS database.
We used facility-level data from Mar. 1, 2018, to Aug. 1, 2020, and to May 31, 2021, for 4 Toronto Central Local Health Integration Network academic hospitals (Kensington Vision and Research Centre, Mount Sinai Hospital, Sunnybrook Health Sciences Centre and Toronto Western Hospital) to capture the details on number of patients and wait times for emergency cases (priority 1) undergoing surgery. These centres consist of hospital-based and stand-alone centres that represent the variety of ophthalmic surgical centres across Ontario.

Lastly, we consulted guidelines from the quality-based procedures for subspecialty surgery from the Ministry of Health and the Provincial Vision Task Force, ${ }^{16}$ the literature and expert opinion from ophthalmology specialists to determine an order of priority based on urgency for vitreoretinal surgery, ${ }^{17}$ glaucoma, cornea, ${ }^{18}$ cataract surgery, ${ }^{16}$ oculoplastics and adult strabismus surgery. Quality-based procedures as a part of Ontario's Health System Funding Reform are clinically related diagnoses and treatments that have been identified using an evidence-based framework as providing opportunity for process improvements, clinical redesign, improved patient outcomes, enhanced patient experience and potential health system cost savings. ${ }^{16}$ For example, urgent vitreoretinal surgical cases were given a higher priority than urgent oculoplastics cases with an acceptable wait time of less than a week before deterioration based on the current literature on decline in functional outcomes of macula sparing and involving retinal detachments. ${ }^{19-22}$ A summary of the parameters used in the model for each of the subspecialties and urgency levels is outlined in Appendix 2, Supplemental Table 2, available at www. cmajopen.ca/content/9/4/E1063/suppl/DC1.

\section{Model structure}

The time-steps of the microsimulation model were each 1 day long. The subspecialties included in the model were cataract surgery (cataract and combination cataract and other procedures), retina surgery (vitrectomy and other vitreoretinal surgery), glaucoma surgery (glaucoma filter or seton and other glaucoma surgeries), corneal surgery (corneal transplant and other cornea surgery), oculoplastics and adult strabismus surgery, each based on their specific characteristics. On initiation of simulations, individuals represent the existing wait-list as of Mar. 15, 2020, based on real data entered in the model. On each subsequent day, estimates of new urgent, and semiurgent or nonurgent cases informed by real data from 2019 to 2020 entered the model and were added to the surgical wait-list (Figure 1).

On each day, a fixed number of procedures were available, informed by the number of procedures completed per month in Ontario based on real administrative data. The surgeries occurred 7 days of the week to account for urgent surgeries after hours and on weekends. Patients with the highest urgency (priority 1) underwent subspecialty surgery, followed by semiurgent groups (priority 2 and 3 ) and then nonurgent groups (priority 4) if the necessary resources were available. Within each urgency level (the urgent, semiurgent and nonurgent classifications), the prioritization for allocation of surgery 
was further broken down to multiple levels (1A, 1B, 2, 3, 4A and $4 \mathrm{~B}$ ) based on length of wait time. Patients remained on the wait-list until the next available resource for surgery became available.

After each surgical intervention, patients were assigned a probability for full recovery with "no further surgical management" required. The remaining patients in the "additional surgery required" category underwent prespecified 2-step surgery (e.g., silicone oil removal and intraocular lens insertion for patients left aphakic after the initial surgery) or repeat surgery for those with a suboptimal surgical outcome after the initial surgery (e.g., intraocular lens repositioning).

Similar to real life, it was assumed that each person could have a maximum 3 surgeries, as repeating more than 3 surgeries for the same condition is extremely rare. Adult strabismus surgery, which may be performed as bilateral surgery for most cases, was captured in the model. For the remaining surgeries, bilateral surgery is much less common, and as such, the model assumed that all other surgeries performed were unilateral. Bilateral cataract surgery represents only $2 \%$ of all cataract cases in Ontario. ${ }^{23}$ Patients moved up through the urgency prioritization levels on the basis of time on the wait-list to account for deterioration of vision status with long delays in surgical repair.

\section{Backlog trajectory and recovery plan}

Simulations started on Mar. 15, 2020, with an end date on Mar. 1, 2023. Outcomes were accrued over a time horizon of 3 years $(35.5 \mathrm{mo})$. For the pandemic phase (base case), representing the backlog created as a result of the COVID-19 pandemic shutdowns in the province (first [March 2020] and third [April 2021] waves), we used the number of available resources for January 2019 to May 2021 from the WTIS database. To reflect hospital resource availabilities for ophthalmic surgery, the model was set up with specific constraints (set number of surgeries and operating room available). The number of new patients awaiting surgery was calculated based on historical numbers from Jan. 1, 2019, in the WTIS database up to Nov. 1, 2020. After Nov. 1, 2020, the number of new patients added to the wait-list and the surgical resources was based on the disease incidence, previous annual growth of the wait-list and Government of Ontario population projections (1.3\% increase in 2021 and $1.4 \%$ increase in 2022). ${ }^{24}$ Given that we expected that the Ontario health care system is efficient and works at $100 \%$ capacity, past volumes would be an indication of available health care resources.

For the postpandemic recovery phase, we estimated the resources required to clear the backlog of patients accumulated on the wait-list since Mar. 15, 2020, for different time horizons.

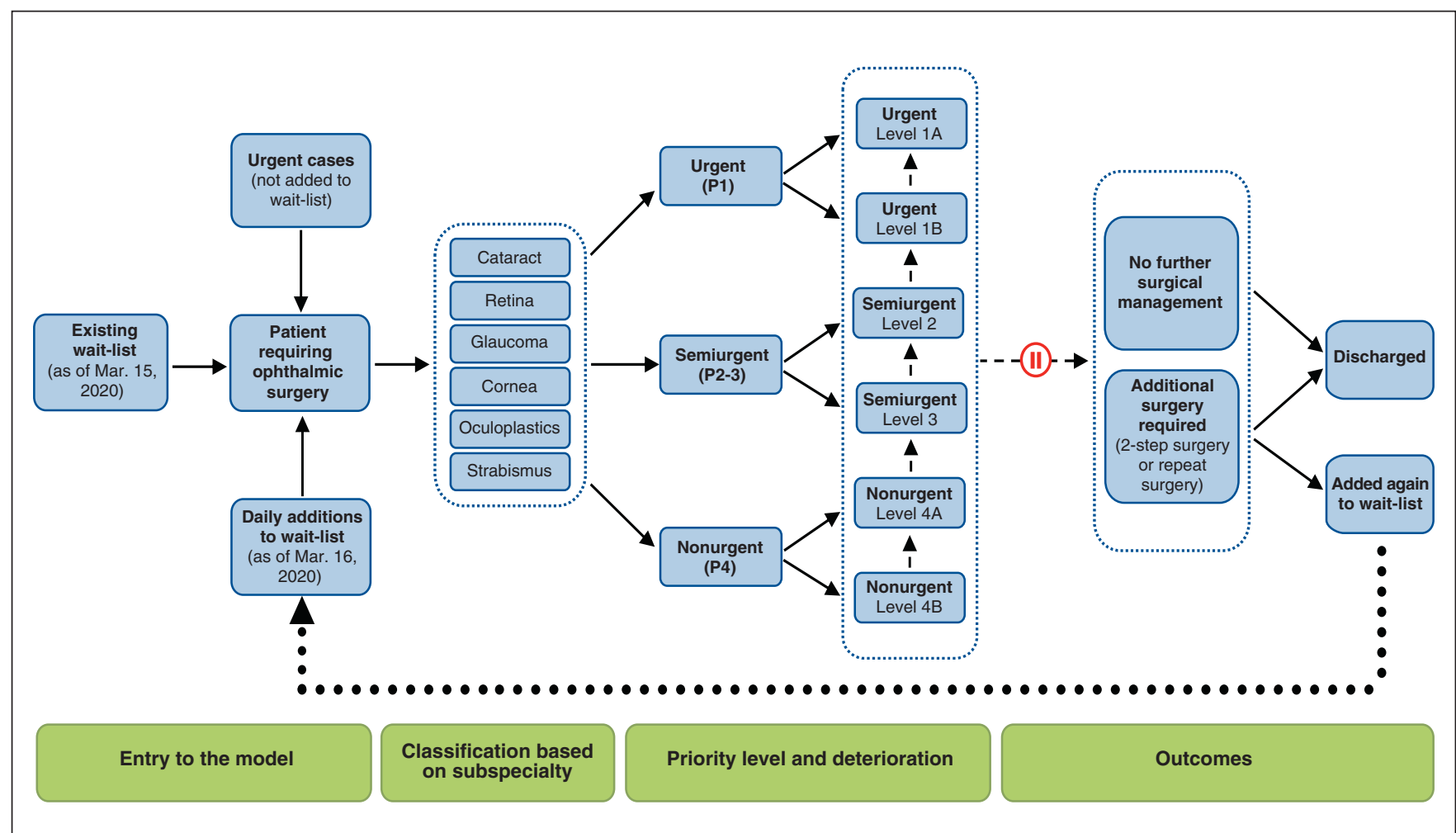

Figure 1: Model schematic depicting patient flow for cases requiring subspecialty ophthalmic surgery. Two entry streams for patients include urgent cases and surgical wait-list (consists of existing wait-list before the pandemic and daily additions following declaration of the pandemic). The stop node (red symbol) represents resource constraint for ophthalmic subspecialty surgery. For patients in semiurgent and nonurgent classifications, there is a deterioration and increase in urgency priority (as indicated by the dashed line) for surgery as the maximum wait time is reached (highest priority given to "Level 1"). This was done to account for the risk of vision impairment associated with delays in surgical repair. Patients move to the "Outcomes" health states after surgery only when resources become available. Those requiring additional surgical interventions will re-enter the model (as indicated by the dotted line). Note: P1-4 = priority level 1-4. 


\section{Model validation}

As the first step to model validation, we verified face validity of the model by consulting practising ophthalmologists in the relevant subspecialties. Debugging was undertaken by verifying the modelling steps and structure for disease trajectories, checking each of the equations used to calculate parameter values, and reviewing the calculations to ensure accuracy of the inputted data. Internal validation of the model involved comparison of intermediate outcomes from the model to observed data from which parameter estimates were obtained.

Next, to confirm the validity of the model outputs, we compared the estimates of wait time obtained from the model for March to November 2019 with the historical data from the WTIS database for the same period. This was done to ensure that the model could adequately predict future wait times for patients awaiting surgery. A comparison of the projected wait times from the model for the months of March to November 2019 showed similarity in the findings between the model and historical data from the WTIS database (124.4, SD 70.8 v. 122.28, SD 134.6, d). These findings support the accuracy of the model in projecting the backlog as a result of the pandemic for 2021-2023.

The model was also run for 2 years after Nov. 1, 2020, in a scenario analysis without pandemic shutdowns. This was done to determine the stability and robustness of the model esti- mates over longer time horizons without the effect of the pandemic. The model projections for wait times for the period November 2020 to November 2022 was 121.5 (SD 102.1) days for all ophthalmologic surgeries. Comparing these findings to real data from March to November 2019, there was no evidence to suggest that the model over- or underestimated the predicted outcomes over longer time horizons when compared with prepandemic wait times.

\section{Statistical analysis}

To account for the variability and uncertainty in the inputs, we conducted a probabilistic sensitivity analysis (PSA) as per CADTH guidelines. ${ }^{13}$ The PSA was run 50 times for each scenario model with about 240000 patients over each year. The PSA incorporates variability in the parameters at 2 levels: patient characteristics, and surgical parameters based on the probability distribution assigned to each parameter. A value is then randomly drawn from the distribution for each model simulation. This process is then repeated many times to derive mean estimates. Each of the 50 scenarios run by the model produces a different result, but over a large number of simulations, the results converge to the average result from a deterministic model. The robustness of the findings despite stochasticity of the parameters is depicted by the clustering of the various scenarios, which suggests that variations in various parameters do

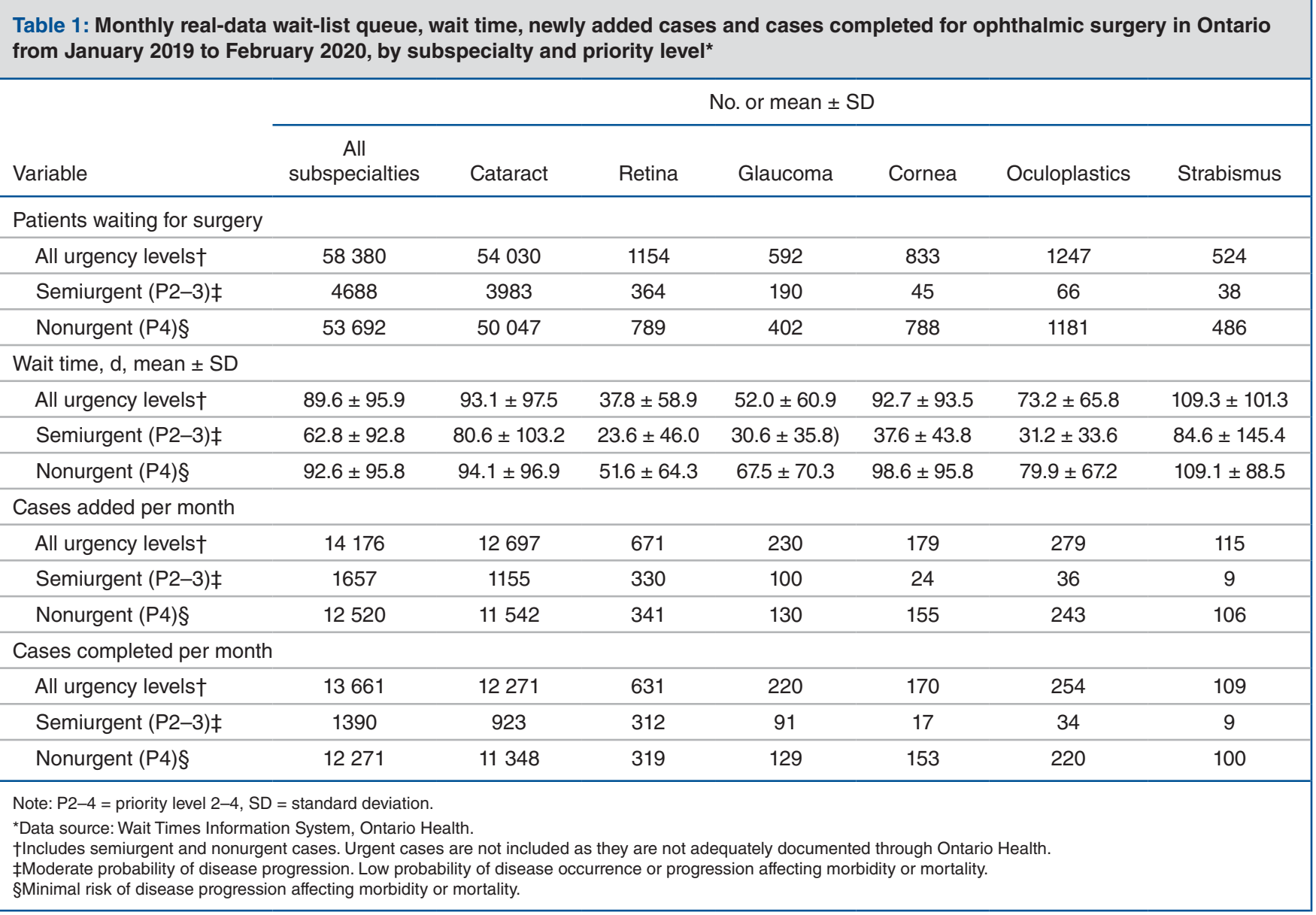


not alter the overall findings of the model (Appendix 3, Supplementary Figure 1, available at www.cmajopen.ca/content/9/4/ E1063/suppl/DC1).

All parameters were assigned a $\beta$ distribution for values bounded by 0 and 1 (probabilities of outcomes), and normal distribution with SD for continuous variables (e.g., wait times in days). The outcomes for all trials were calculated as a mean and SD. All modelling and analyses were conducted using TreeAge Pro 2021 (TreeAge Software). The mean output from the microsimulation was visualized using the $\mathrm{R}$ statistical program (version 4.0.4).

\section{Ethics approval}

Ontario Health collects personal health information as part of the WTIS pursuant to its prescribed entity authority under section 45 of the Personal Health Information Protect Act, 2004. Ontario Health provided aggregate deidentified data from WTIS to construct the model.

\section{Results}

As of Mar. 15, 2020, a total of 56923 patients were on the wait-list in the province of Ontario awaiting non-emergency ophthalmic surgery. On average, the monthly number of nonemergency cases for January 2019-February 2020 added to the wait-list was 14176 , and a monthly average of 13659 patients underwent non-emergency surgery. A summary of the monthly wait-list queue, surgical throughputs, newly added cases and wait times for ophthalmic surgery in Ontario for January 2019-February 2020 is presented in Table 1 . The number of non-emergency surgeries performed in the province decreased by $45 \%, 98 \%$ and $97 \%$ in March, April and May 2020 (first wave), respectively, compared with the same months in 2019. The number of non-emergency surgeries performed in the province decreased by $48 \%$ and $80 \%$ in April and May 2021 (third wave), respectively, compared with the same months in 2019. Figure 2 shows the surgical throughput during the pandemic phase compared with historical data from 2019.

\section{Model projections}

The total number of patients awaiting surgery 1 year after the pandemic began increased by $112 \%$ (62 503 additional cases) in February 2021 compared with February 2020. By 2 years, the overall estimated number of patients awaiting surgery grew by $129 \%$ from February 2020 . More specifically, the estimated number of patients awaiting cataract, vitreoretinal, glaucoma, cornea, oculoplastics and strabismus surgeries grew by $140 \%, 133 \%, 171 \%, 175 \%, 115 \%$ and $340 \%$, respectively, at 2 years after the pandemic

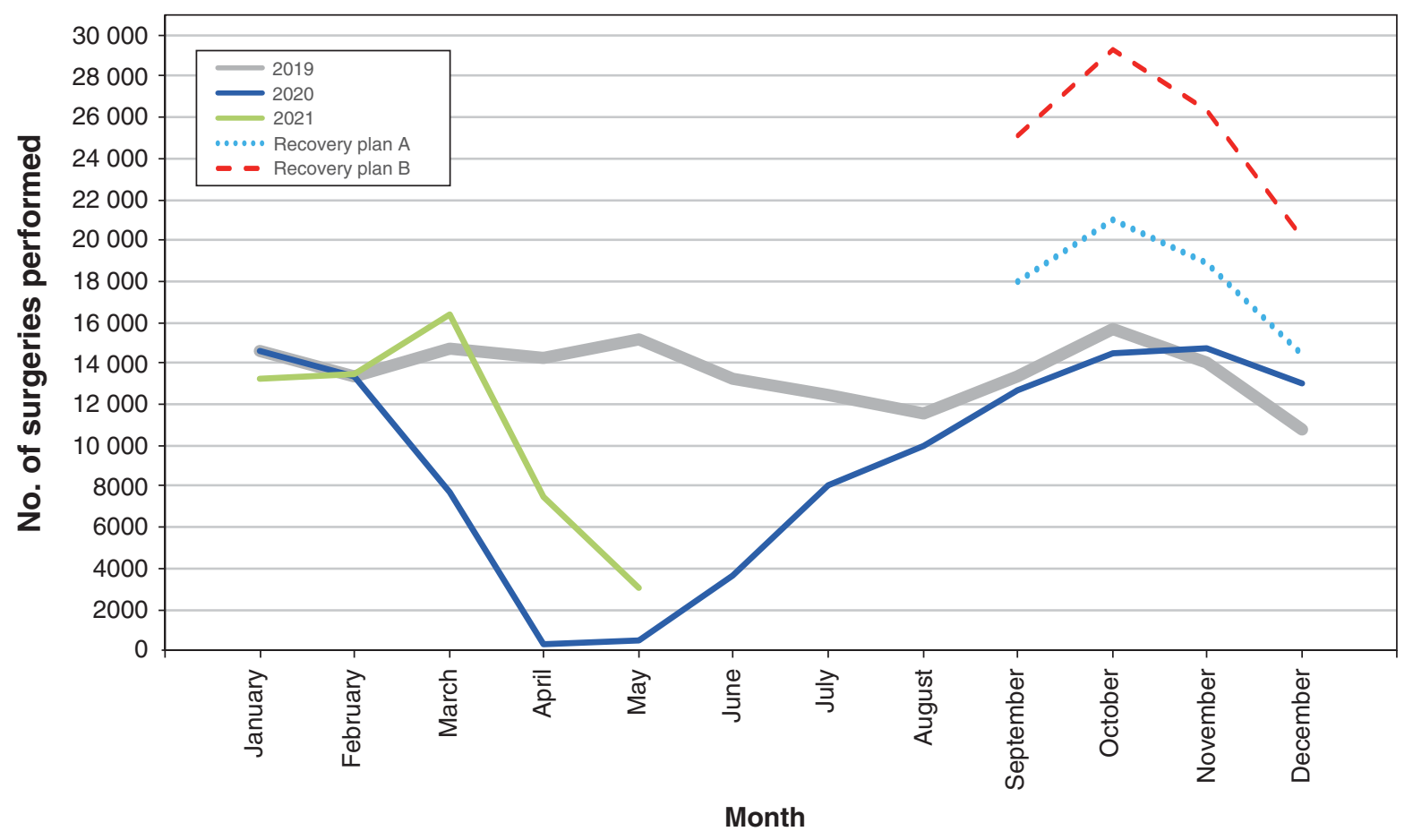

Figure 2: Monthly surgical throughputs based on real data after the pandemic began (solid blue line = 2020; solid green line = 2021) compared with 2019 (solid grey line). The dotted line shows the model-estimated monthly increase in number of surgeries required to clear the backlog created as a result of COVID-19 over a 2-year period starting in September 2021 (recovery plan A). The dashed line shows the monthly increase in number of surgeries required to clear the backlog over a 1-year period starting in September 2021 (recovery plan B). These recovery plan results show the degree of escalation in resource availability required to return to the prepandemic wait-list queue and wait times for ophthalmic surgery. Note that only the first months of the recovery plans are depicted in the graph. 
began. By 3 years, the overall estimated number of patients awaiting surgery grew by $150 \%$ from February 2020. Provincial estimates of the backlog size by surgical subspecialty type over 3 years after the pandemic began are presented in Figure 3. Overall, the growth in the backlog as a result of the number of patients awaiting surgery was driven by the volume of nonurgent cases (Appendix 4, Supplemental Figure 2, available at www.cmajopen.ca/content/9/4/ E1063/suppl/DC1).
The mean wait time for patients for all subspecialty surgeries increased to an estimated 282 (SD 91) days in March 2023 compared with 94.4 (SD 97.4) days in 2019 (Table 2). The estimated time to surgery for the initial patients on the wait-list at the start of the pandemic was 197.3 (SD 95.1) days. Of the 56047 patients on the wait-list for semiurgent and nonurgent surgery at the start of the pandemic, the results suggested that $99 \%$ had surgery within 12 months (Appendix 5, Supplemental Figure 3, available at www.cmajopen.ca/content/9/4/E1063/suppl/DC1).
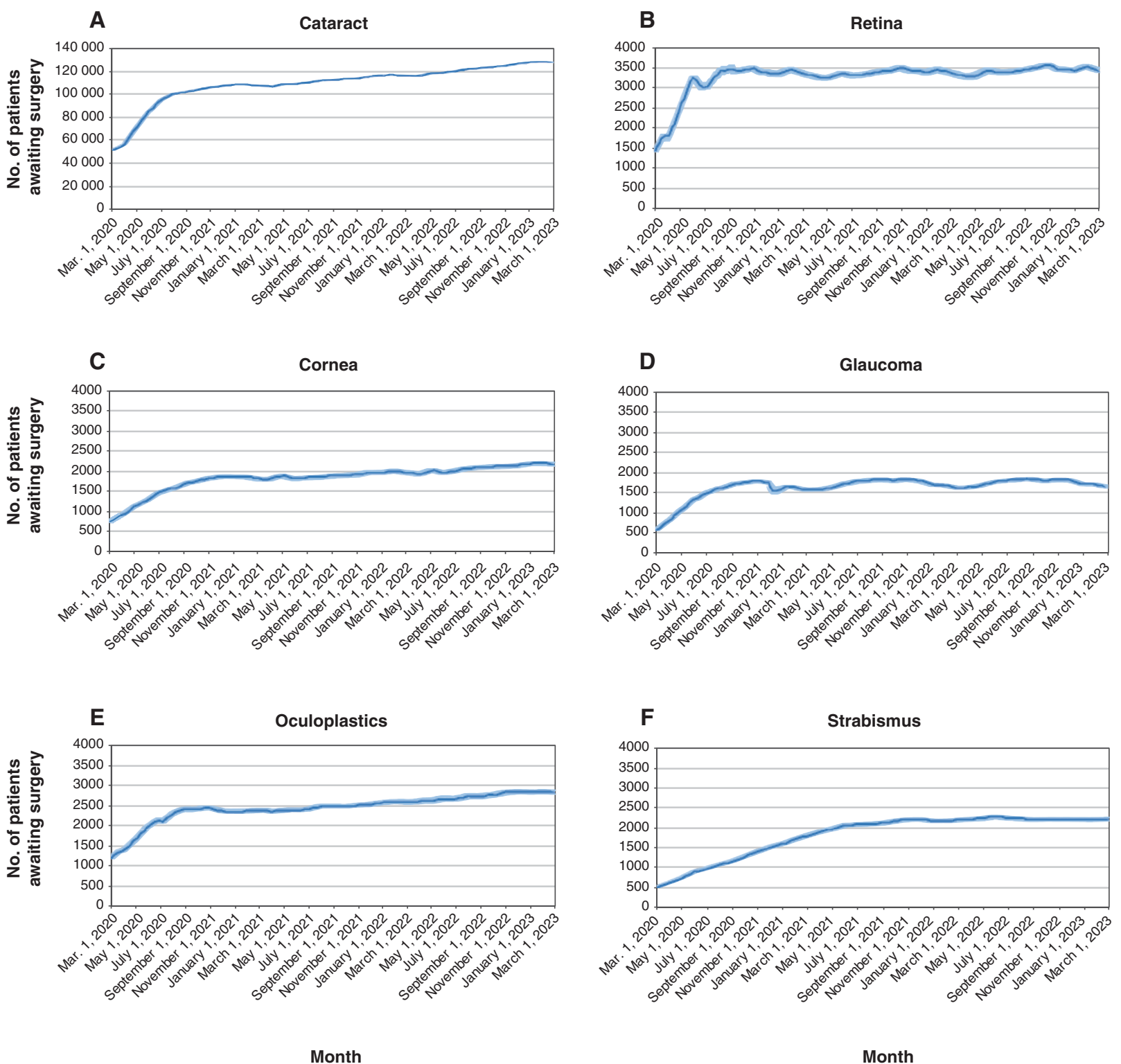

Figure 3: Monthly model-estimated accumulation of patients awaiting surgery for all ophthalmic surgeries and subspecialty types, including cataract surgery ( $\mathrm{A}$, cataract and combination cataract and other procedures), retina surgery ( $\mathrm{B}$, vitrectomy and other vitreoretinal surgery), corneal surgery ( $C$, corneal transplant and other cornea surgery), glaucoma surgery (D, glaucoma filter or seton and other glaucoma surgeries), oculoplastics (E) and adult strabismus surgery (F) from March 2020 to March 2023. The simulations were run 50 times (variations in projected estimated represented by lighter blue lines) for a total of 240000 patients. Note that the $y$-axis scale for cataract surgery (A) is different than that of the other subspecialty groups. 
Regarding backlog clearance, the increase in provincial monthly resources required to clear all surgery types by March 2023 was estimated to be 34\% (4626 additional surgeries per month, proposed recovery plan A) if starting in September 2021. Comparatively, recovery to the prepandemic wait-list by March 2022 would require an increase of $87 \%$ (11838 additional surgeries per month, proposed recovery plan B) if starting in September 2021 (Figure 2).

\section{Interpretation}

Our findings show that the magnitude of the ophthalmic surgical backlog from the COVID-19 pandemic has important implications for the recovery phase.

After Mar. 15, 2020, the pandemic shutdowns resulted in reduction of surgical volumes for several months and an associated increase in the number of patients awaiting surgery. Similarly, drastic reductions by $77 \%-90 \%$ of the usual volume of surgeries performed have been noted at other tertiary ophthalmic surgical centres in North America $^{25}$ and Europe. ${ }^{26}$ Despite the gradual recovery in surgical activity, without any substantial increases in resources to support the backlog of surgical cases, incoming new urgent cases will lead to further delays in surgeries for semiurgent and nonurgent cases. In addition to modelling the projected backlog as a result of the pandemic, we forecast potential recovery planning scenarios. Our model estimates ophthalmology resource use and availability based on priority level for each subspecialty surgery, while taking into account the increasing urgency over time owing to the deterioration expected with delayed access to care. The validity of our study is strengthened by the incorporation of historical data from the WTIS database and the local health care resources estimates of potential capacity, as well as the use of evidence-based guidelines on established prioritization for subspecialty surgery.
One of the important considerations in the context of delay to surgery is the deterioration in vision outcomes in patients who are awaiting surgery. For the patients on the wait-list at the time of the pandemic, on average, there was a delay of 197 days until surgery. With the increase in the number of surgical procedures performed during the postpandemic recovery phase, there will be a progressive plateau of the number of patients awaiting surgery on the wait-list; however, it is important to note that nonurgent and semiurgent cases exceeding the acceptable wait times for surgery may progressively deteriorate and become urgent. For example, the patients awaiting subspecialty surgery for the retina had an average of 48 days of wait time immediately after the pandemic began, with a substantial increase to 121 and 124 days at 2 and 3 years, respectively, after the start of the pandemic. These progressive increases in wait time for nonurgent and semiurgent cases, such as elective epiretinal membrane peel or macular hole repair, may lead to consequent vision loss or more challenging surgical repairs. ${ }^{27,28}$ Within the realm of other common procedures, recent studies have also reported an average delay of 5.34 weeks as a result of COVID-19 lockdowns for patients requiring intravitreal injections. ${ }^{29}$ The reported implications of this delay were more profound vision loss in patients with diabetic macular edema, proliferative diabetic retinopathy and retinal vein occlusion. ${ }^{29}$

As shown by our study, among all subspecialty ophthalmology surgeries, cataract surgery represents the highest volume of cases (12697) added to the wait list each month. Aside from the effects of the pandemic, there is an increasing need for higher volumes of cataract surgeries performed with the growth and aging of the population. A recent report by the Ontario Medical Association suggested that, on comparison of billings to the Ontario Health Insurance Plan in 2020 and the same period in 2021, the estimated backlog for cataract surgery was the third highest among all other procedures in Ontario. ${ }^{30}$ Hatch and colleagues projected a minimum

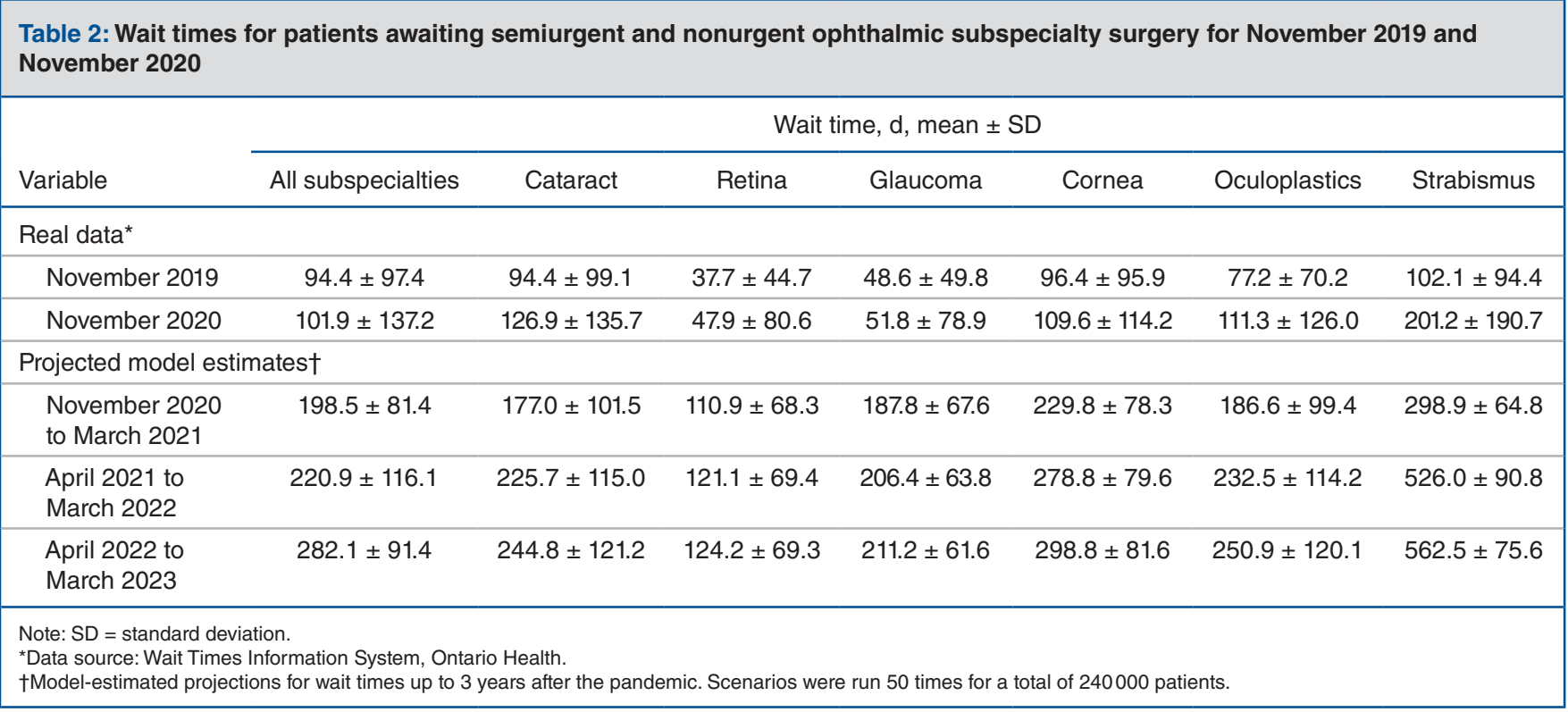


increase of $128 \%$ in surgical volumes, or about 4.3 million additional cataract surgeries required per year for 2036 in North America. ${ }^{31}$ Delays in semiurgent and nonurgent procedures, such as cataract surgery, not only have implications on quality of life, ${ }^{32}$ but also have been shown to be associated with increased falls in patients awaiting surgery. ${ }^{33}$ The psychological and physical traumas associated with increased wait times have also been noted by studies on other surgical subspecialty care. ${ }^{34}$ With consideration of the implications of delay in surgery and economic benefits, authors have advocated for bilateral surgeries ${ }^{35}$ and combined procedures such as phacovitrectomy. ${ }^{36}$

In addition to the delay to surgery while a patient is on the wait-list, there is an inherent delay in presentation to specialized surgical care. ${ }^{37-39}$ These delays are shown to be further exacerbated by the COVID-19 pandemic and patient hesitation to seeking care in the ophthalmology setting. ${ }^{6,7}$ In the model in our study, the number of adult patients awaiting surgical intervention for strabismus grew notably by $340 \%$ within 2 years. Adult strabismus surgery is rarely considered to be of urgent priority compared with other ophthalmic subspecialty surgery. Nonetheless, this patient population already experiences delays in presentation, with about 20 years from time of onset to seeking surgical intervention. ${ }^{40}$ As such, further delays for strabismus surgery as a result of the pandemicinduced surgical backlog may result in social, psychological and economic burdens for patients. ${ }^{41}$

With the rapidly evolving nature of the pandemic and its unpredictable impact on the health care system, it is paramount that decision-makers and government representatives continue using models as tools for evidence generation in support of the policy decision-making processes. ${ }^{9}$ Previous models by our group have guided decisions regarding the estimation of pandemic-induced depletion of hospital resources, ${ }^{10}$ as well as policies for transmission risk in schools versus community-based settings. ${ }^{42}$ Other models on elective vascular surgical delays caused by COVID-19 have estimated an 8-month recovery period to achieve a steady state in the number of patients awaiting surgery. ${ }^{43}$ Additionally, we have estimated incremental growth in the wait-list for all cardiac procedures during the COVID-19 pandemic and the implications for the provision of cardiovascular care. ${ }^{44}$ Wang and colleagues estimated an additional 719 hours of weekly operating room time to clear the backlog created as a result of the pandemic for surgical specialties such as ophthalmology, gynecology, general surgery, orthopedics and urology. ${ }^{1}$ The model by Wang and colleagues, however, did not investigate ophthalmology in detail and underestimated the impact of continued delay on vision outcomes of patients. ${ }^{43}$

Projections of required resources for the future are essential for introducing policies such as implementation of operating room hours on weekends and extended hours on weekdays. Our projected model estimates suggest that a planned increase in provincial monthly resources of at least $34 \%$ will be required to return to the prepandemic surgical backlog by March 2023, while care is also provided for the normal flux of surgical patients. To provide context, a ramp-up of $34 \%$ in surgical volumes may be equivalent to supplementing a typical 8-hour day or a 40-hour week with an additional 2 hours added to 2 working weekdays, as well as 1 full weekend workday for surgical cases. The speed of recovery could be accelerated if hospitals were to share the burden of cases across the province. Other considerations such as the operational directives regarding patient transport, operating room preparation, personnel dressing and environmental sanitization should be established to improve efficiency of the surgeries performed. ${ }^{45,46}$

One of the major strengths of this microsimulation model is the use of comprehensive databases that accurately captured the impact of the pandemic on surgical centres. Furthermore, we had access to detailed information on cases from several facilities. Modelling is a validated and useful tool for providing evidence to support policy-makers and decision-making throughout a pandemic. ${ }^{9}$ Our validation results provided strong support that the current model estimates are comparable to historical data. The complex dynamic navigation of patients and interaction between resource availability and demand from the population for the future is most accurately estimated using microsimulations such as in this model. This model can be used for other scenarios, with new interruptions in ophthalmic surgeries. With the concerns of additional lockdowns in the future, it is important to note the competing resource allocation for various other procedures and surgical specialties. $^{4,47,48}$

\section{Limitations}

This model relies on forecasting COVID-19 cases based on historical data, current evidence-based guidelines and assumptions of patterns in surgical practice. Actual data on the number of patients awaiting surgery beyond March 2020 is currently not available owing to the reductions in number of patients seeking health care during the pandemic. Although we aimed to capture the population growth rates of $1.3 \%-1.4 \%$ when estimating the number of patients presenting for each subspecialty surgery based on historical data, we did not take into consideration other factors such as aging population and evolving changes in health care needs over time, which could underestimate the backlog. This model also assumed that all reported historical surgical volumes were appropriately indicated for surgery, which may not always hold true depending on variations in practice patterns. ${ }^{49}$

Bilateral cataract surgeries may be performed, though less commonly; however, the model was set up to simulate each patient eye separately, which may overestimate the resources required to clear the backlog. Data for urgent cases was based on tertiary care centres in the Greater Toronto Area, which likely provides a skewed number of emergencies. Lastly, it is expected that predictions over longer time horizons will be progressively less robust in their reliability. A review of the impact of the wait times on patient quality of life was outside the scope of this study but may be studied in future models. 


\section{Conclusion}

The findings from this microsimulation model informed by historical provincial data of surgical volumes depicts the projected wait time for surgery and growing wait list for ophthalmology subspecialty surgery from the COVID-19 pandemic. Our projections suggest that a ramp-up in surgical volumes will be needed to return the backlog to prepandemic levels. The proposed recovery plans will aid jurisdictions in optimizing their response to the evolving needs of the population for vision-saving surgeries.

\section{References}

1. Wang J, Vahid S, Eberg M, et al. Clearing the surgical backlog caused by COVID-19 in Ontario: a time series modelling study. CMAF 2020;192: E1347-56.

2. Wu K, Smith CR, Lembcke BT, et al. Elective surgery during the COVID-19 pandemic. NEngl f Med 2020;383:1787-90.

3. COVIDSurg Collaborative. Elective surgery cancellations due to the COVID19 pandemic: global predictive modelling to inform surgical recovery plans. $\mathrm{Br}$ 7 Surg 2020;107:1440-9.

4. Søreide K, Hallet J, Matthews JB, et al. Immediate and long-term impact of the COVID-19 pandemic on delivery of surgical services. Br F Surg 2020;107: 1250-61.

5. A measured approach to planning for surgeries and procedures during the COVID-19 pandemic. Toronto: Ontario Health; 2020. Available: https:// www.corhealthontario.ca/OH_Resuming_Surg_Proc-(May-13).pdf (accessed 2020 June 3).

6. Patel LG, Peck T, Starr MR, et al. Clinical presentation of rhegmatogenous retinal detachment during the COVID-19 pandemic: a historical cohort study. Ophthalmology 2021;128:686-92.

7. Romano F, Monteduro D, Airaldi M. Increased number of submacular hemorrhages as a consequence of COVID-19 lockdown. Opbthalmol Retina 2020;4: 1209-10.

8. Shelton BA, Berdahl G, Sawinski D, et al. Optimal timing of hepatitis C treatment among HIV/HCV coinfected ESRD patients: pre- vs posttransplant. $\mathrm{Am}$ 7 Transplant 2019;19:1806-19.

9. Mac S, Mishra S, Ximenes R, et al. Modeling the coronavirus disease 2019 pandemic: a comprehensive guide of infectious disease and decision-analytic models. 7 Clin Epidemiol 2021;132:133-41.

10. Barrett K, Khan YA, Mac S, et al. Estimation of COVID-19-induced depletion of hospital resources in Ontario, Canada. CMA7 2020;192:E640-6.

11. Time to patient's first eye specialist appointment. Toronto: Health Quality Ontario. Available: https://www.hqontario.ca/System-Performance/Wait-Times -for-Surgeries-and-Procedures/Wait-Times-for-Eye-Surgeries-including-Cataract -Surgery/Time-to-Patients-First-Eye-Specialist-Appointment (accessed 2020 Dec. 27)

12. Hodge WG, Ramsay T, Fergusson D, et al. An investigation of the relationship between cataract surgery wait times and rates of surgery. Can 7 Ophthalmol 2012;47:11-5.

13. Guidelines for the economic evaluation of health technologies: Canada. 4th ed. Ottawa: Canadian Agency for Drugs and Technologies in Health (CADTH); 2017.

14. Husereau D, Drummond M, Petrou S, et al.; ISPOR Health Economic Evaluation Publication Guidelines-CHEERS Good Reporting Practices Task Force. Consolidated health economic evaluation reporting standards (CHEERS) explanation and elaboration: a report of the ISPOR health economic evaluation publication guidelines good reporting practices task force. Value Health 2013; 16:231-50

15. Population estimates, quarterly. Ottawa: Statistics Canada. Available: https:// www150.statcan.gc.ca/t1/tbl1/en/tv.action?pid=1710000901 (accessed 2020 Dec. 29).

16. Quality-based procedures clinical handbook: cataract day surgery. Toronto: Ministry of Health and Long-Term Care; 2021. Available: www.health.gov. on.ca/en/pro/programs/ecfa/docs/qbp_cataract.pdf (accessed 2020 Dec. 28).

17. Quality-based procedures clinical handbook for integrated retinal care. Toronto: Ministry of Health and Long-Term Care; 2014.

18. Quality-based procedures clinical handbook for integrated corneal transplant care. Toronto: Ministry of Health and Long-Term Care; 2018. Available: https://www.health.gov.on.ca/en/pro/programs/ecfa/docs/hb_corneal.pdf (accessed 2020 Dec. 28).

19. Greven MA, Leng T, Silva RA, et al. Reductions in final visual acuity occur even within the first 3 days after a macula-off retinal detachment. Br 7 Ophthalmol 2019;103:1503-6.

20. van Bussel EM, van der Valk R, Bijlsma WR, et al. Impact of duration of macula-off retinal detachment on visual outcome: a systematic review and meta-analysis of literature. Retina 2014;34:1917-25.
21. Ehrlich R, Niederer RL, Ahmad N, et al. Timing of acute macula-on rhegmatogenous retinal detachment repair. Retina 2013;33:105-10.

22. Frings A, Markau N, Katz T, et al. Visual recovery after retinal detachment with macula-off: Is surgery within the first $72 \mathrm{~h}$ better than after? $\mathrm{Br} 7$ Ophthalmol 2016;100:1466-9.

23. Grzybowski A, Wasinska-Borowiec W, Claoué C. Pros and cons of immediately sequential bilateral cataract surgery (ISBCS). Saudi 7 Ophthalmol 2016; 30:244-9.

24. Ontario population projections. Toronto: The Government of Ontario. Available: https://www.fin.gov.on.ca/en/economy/demographics/projections/ (accessed 2020 Dec. 28).

25. Al-Khersan H, Kalavar MA, Tanenbaum R, et al. Emergent ophthalmic surgical care at a tertiary referral center during the COVID-19 pandemic. Am 7 Ophthalmol 2021;222:368-72.

26. dell'Omo R, Filippelli M, Semeraro F, et al. Effects of the first month of lockdown for COVID-19 in Italy: a preliminary analysis on the eyecare system from six centers. Eur 7 Ophthalmol 2020 Aug. 24 [Epub ahead of print]. doi: 10.1177/1120672120953074.

27. Yun C, Oh J, Hwang S-Y, et al. Morphologic characteristics of chronic macular hole on optical coherence tomography. Retina 2012;32:2077-84.

28. Gomes NL, Corcostegui I, Fine HF, et al. Subfoveal pigment changes in patients with longstanding epiretinal membranes. Am 7 Ophthalmol 2009;147: $865-8$.

29. Song $W$, Singh RP, Rachitskaya AV. The effect of delay in care among patients requiring intravitreal injections. Ophthalmol Retina 2021 Jan. 1 [Epub ahead of print]. doi: 10.1016/j.oret.2020.12.020.

30. OMA estimates pandemic backlog of almost 16 million health-care services [news release]. Toronto: Ontario Medical Association; 2021 June 9. Available: https://www.oma.org/newsroom/news/2021/jun/oma-estimates-pandemic -backlog-of-almost-16-million-health-care-services/ (accessed 2021 June 9).

31. Hatch WV, de L Campbell E, Bell CM, et al. Projecting the growth of cataract surgery during the next 25 years. Arch Ophthalmol 2012;130:1479-81.

32. Lamoureux EL, Fenwick E, Pesudovs K, et al. The impact of cataract surgery on quality of life. Curr Opin Ophthalmol 2011;22:19-27.

33. Chen CL, McLeod SD, Lietman TM, et al. Preoperative medical testing and falls in medicare beneficiaries awaiting cataract surgery. Ophthalmology 2021; 128:208-15.

34. Strong SM, Magama Z, Mallick R, et al. Waiting for myomectomy during the COVID-19 pandemic: the vicious cycle of psychological and physical trauma associated with increased wait times. Int 7 Gynaecol Obstet 2020;151:303-5.

35. Ahmed IIK, Hill WE, Arshinoff SA. Bilateral same-day cataract surgery: an idea whose time has come \#COVID-19. Opbthalmology 2021;128:13-4.

36. Antaki F, Milad D, Javidi S, et al. Vitreoretinal surgery in the post-lockdown era: making the case for combined phacovitrectomy. Clin Ophthalmol 2020;14: 2307-9.

37. Liddy C, Moroz I, Affleck E, et al. How long are Canadians waiting to access specialty care? Retrospective study from a primary care perspective. Can Fam Physician 2020;66:434-44.

38. Liddy C, Nawar N, Moroz I, et al. Understanding patient referral wait times for specialty care in Ontario: a retrospective chart audit. Healthc Policy 2018;13: 59-69.

39. Lindeke-Myers A, Zhao PYC, Meyer BI, et al. Patient perceptions of SARS$\mathrm{CoV}-2$ exposure risk and association with continuity of ophthalmic care. 7AMA Ophthalmol 2021;139:508-15.

40. Coats DK, Stager DR, Beauchamp GR, et al. Background: reasons for delay of surgical intervention in adult strabismus. Arch Ophthalmol 2005;123:497-9.

41. Al Shehri F, Duan L, Ratnapalan S. Psychosocial impacts of adult strabismus and strabismus surgery: a review of the literature. Can $\mathcal{F}$ Ophthalmol 2020;55: 445-51.

42. Naimark D, Mishra S, Barrett K, et al. Simulation-based estimation of SARS$\mathrm{CoV}-2$ infections associated with school closures and community-based nonpharmaceutical interventions in Ontario, Canada. FAMA Netw Open 2021;4: e213793.

43. Brown CS, Albright J, Henke PK, et al. Modeling the elective vascular surgery recovery after COVID-19: implications for moving forward. 7 Vasc Surg 2021; 73:1876-80.e1.

44. Tam DY, Naimark D, Natarajan MK, et al. The use of decision modelling to inform timely policy decisions on cardiac resource capacity during the COVID-19 pandemic. Can 7 Cardiol 2020;36:1308-12.

45. Coccolini F, Perrone G, Chiarugi M, et al. Surgery in COVID-19 patients: operational directives. World f Emerg Surg 2020;15:25.

46. Rozario N, Rozario D. Can machine learning optimize the efficiency of the operating room in the era of COVID-19? Can 7 Surg 2020;63:E527-9.

47. Shiff B, Breau RH, Patel P, et al. Impact of time to surgery and surgical delay on oncologic outcomes for renal cell carcinoma. F Urol 2021;205:78-85.

48. Sienaert P, Lambrichts S, Popleu L, et al. Electroconvulsive therapy during COVID-19-times: our patients cannot wait. Am 7 Geriatr Psychiatry 2020;28: $772-5$.

49. Cooper Z, Sayal P, Abbett SK, et al. A conceptual framework for appropriateness in surgical care: reviewing past approaches and looking ahead to patientcentered shared decision making. Anesthesiology 2015;123:1450-4. 
Affiliations: Department of Ophthalmology and Vision Sciences (Felfeli), University of Toronto; Toronto Health Economics and Technology Assessment (THETA) Collaborative (Ximenes), University Health Network; Sunnybrook Health Sciences Centre (Naimark), Toronto, Ont.; Ivey Eye Institute (Hooper), Western University, London, Ont.; Department of Ophthalmology (Campbell), Queen's University, Kingston, Ont.; Kensington Vision and Research Centre (El-Defrawy), Kensington Eye Institute, and Institute of Health Policy, Management and Evaluation (Sander), University of Toronto, Toronto, Ont.

Contributors: Tina Felfeli, Raphael Ximenes, David Naimark, Sherif ElDefrawy and Beate Sander conceived and designed the study. Tina Felfeli, Philip Hooper, Robert Campbell and Sherif El-Defrawy contributed to the acquisition of data. Tina Felfeli, Raphael Ximenes, David Naimark and Beate Sander contributed to the data analysis. Tina Felfeli, Raphael Ximenes, Sherif El-Defrawy and Beate Sander interpreted the data. Tina Felfeli drafted the manuscript, which all of the authors revised. All of the authors gave final approval of the version to be published and agreed to be accountable for all aspects of the work.

Funding: Tina Felfeli has received grants from Fighting Blindness Canada, the Vanier Canada Graduate Scholarship, Canada Graduate Scholarship - Master's, the Vision Science Research Program, and Postgraduate Medical Education Research Awards as part of postsecondary research funding. This research was supported by COVID-19 Rapid Research Funding (C-291- 2431272-SANDER) through the Ontario Ministry of
Health, Ontario Together grant. This research was also supported, in part, by a Canada Research Chair in Economics of Infectious Diseases grant held by Beate Sander (CRC-950-232429). The funding organizations had no role in the design or conduct of this research.

Content licence: This is an Open Access article distributed in accordance with the terms of the Creative Commons Attribution (CC BYNC-ND 4.0) licence, which permits use, distribution and reproduction in any medium, provided that the original publication is properly cited, the use is noncommercial (i.e., research or educational use), and no modifications or adaptations are made. See: https://creativecommons.org/ licenses/by-nc-nd/4.0/

Data sharing: Portions of the data are available to others and can be accessed by emailing the corresponding authors.

Acknowledgement: Partial aspects of this study were presented at the following virtual meetings: Institute of Health Policy, Management and Evaluation (IHPME) Annual Research Meeting held on Nov. 20, 2020, in Canada; Health Technology Research Annual Meeting held on Feb. 5, 2021, in Brazil; Society for Medical Decision Making North American Meeting, held Oct. 18-20, 2021.

Supplemental information: For reviewer comments and the original submission of this manuscript, please see www.cmajopen.ca/content/9/4/ E1063/suppl/DC1. 